\title{
Folena nel primo Quaderno del Circolo filologico linguistico padovano*
}

\author{
Vittorio Coletti
}

PUBBLICATO: 27 APRILE 2021

I n altri appuntamenti organizzati per ricordare Gianfranco Folena si è ricordata la sua instancabile attività di animatore, promotore e organizzatore di ricerche, studi, scambi culturali, in cui ha fatto da generoso maestro e autorevole sostegno di tanti giovani studiosi. Un angolo da cui osservare questo suo speciale impegno, oltre ovviamente a quello dell'università e dell'Accademia della Crusca, è certamente il Circolo filologico linguistico padovano, da lui fondato e seguito per anni con incessante cura e quasi paterna sollecitudine, dalle sedute settimanali di Padova ai convegni estivi di Bressanone, di cui era, in sostanza, non solo l'ideatore, ma anche il primo e principale ascoltatore e interlocutore e spesso pure l'intrattenitore e la guida turistica dei convegnisti. Il Circolo ha documentato le proprie attività con i suoi celebri Quaderni del Circolo Filologico Linguistico Padovano, poi appoggiati alla rivista trimestrale "Quaderni di Retorica e poetica", e con gli Atti dei numerosi convegni interuniversitari e italo-tedeschi di Bressanone.

Un insieme impressionante, specie se si guarda a quanti sono stati i volumi dei Quaderni o degli Atti prefati da Folena, vale a dire di incontri e iniziative di ricerca da lui direttamente ispirate, promosse, seguite, ospitate. Tra i più antichi Quaderni ricordo il secondo, a più voci, su Lingua e strutture del teatro italiano del rinascimento (I970), il libro di Sergio Raffaelli su Semantica tragica di Federico Della Valle (I973), quello di Gian Piero Brunetta su Forma e parola nel cinema (I970), e poi la serie dei "Quaderni di Retorica e poetica" sul Diario, la Lettera familiare, L'autobiografia, la Lingua scorciata. Detto, motto, aforisma; tra i convegni (e relativi Quaderni) ricordo quelli su Teoria e analisi del testo, su Retorica e politica, Retorica e classi sociali, su Il titolo e il testo e Strategie del testo e sull'Autocommento; tra i convegni italo- tedeschi: Attualità della retorica, Simbolo, metafora, allegoria: un elenco incompleto ma che basta già a dare un'idea della varietà di interessi, della larghezza e liberalità di metodi e temi, dell'ampiezza dell'arco temporale considerato.

Qui vorrei soffermarmi solo sul primo Quaderno del Circolo e sulla sua presentazione da parte di Folena. Siamo di fronte a uno dei testi fondatori della critica formale in Italia. Si tratta delle celebri Ricerche sulla lingua poetica contemporanea, uscite nel I966, con saggi di Fernando Bandini su Rebora, di Lorenzo Polato su Saba, di Pietro Spezzani su Ungaretti, di Pier Vincenzo Mengaldo su Montale e di Anco Marzio Mutterle su Pavese lirico. Con quel libro, se proprio non inizia, si avvia nell'inesplorato dominio dell'attualità letteraria una critica di tipo linguistico della poesia e lo fa con una caratteristica: la concretezza analitica, la storicizzazione, la cautela nella teorizzazione, pur nell'adozione di nuovi metodi, l'attenzione ai significati attraverso l'analisi dei significanti.

Per la verità, fin da subito è stato un tratto chiaro dell'analisi formale italiana l'attenzione alla concretezza individuale (che favoriva l'analisi stilistica) di testi e autori, alla diacronia dei fenomeni linguistici e all'orizzonte storico culturale in cui si situano. Del resto, poco prima delle Ricerche padovane erano usciti Lingua, stile e societá di Cesare Segre (Feltrinelli r963) e Ritmo e melodia nella prosa italiana di Gian Luigi Beccaria (Olschki I964, n.e. 2013), che mettevano a frutto nuove sensibilità e metodologie su corpora letterari storicamente ben definiti e analizzati, senza mai perdere di vista il senso e il contesto. Neppure il saggio più proiettato verso le nuove metodologie, la lettura degli 
Orecchini di Montale di D’Arco Silvio Avalle (Einaudi 1965), forse lo studio più jakobsoniano mai uscito su un testo della nostra letteratura, era ingabbiato dalla teoria e si concentrava con varietà di approcci su un unico, eccezionale testo. Ancora pochi anni dopo, il primo importante libro "formalista" di Maria Corti coniugava i Metodi e Fantasmi (Feltrinelli ig69), la teoria con la storia e la filologia. E a pensarci bene, il fortunato libro sui Metodi attuali della critica in Italia (ERI I970) curato da Corti e Segre, salutato come l'inaugurazione della nuova critica italiana, affiancava ai nuovi metodi formali anche altre metodologie, sancendo subito la salutare assenza di unideologia o di qualsiasi fanatismo formalista tra $\mathrm{i}$ nostri migliori linguisti-critici. La congiunzione di formalismo/strutturalismo e storicismo, di lingua e storia, è una felice combinazione della nostra critica letteraria degli anni sessanta, mai interrotta e ripresa presto anche da chi aveva più sondato l'universo delle teorie negli anni settanta: basti pensare che Maria Corti, dopo Il viaggio testuale (Einaudi I978), torna alla concretezza filologica di Dante e di Cavalcanti, riprendendo il magnifico filo dei suoi precoci, magistrali studi storico-linguistici sulla Sintassi della lingua poetica avanti lo Stilnovo con cui aveva iniziato nel 1953 (Olschki, riediti postumi Galluzzo 2005).

Folena inizia la sua Presentazione riepilogando la breve, allora recentissima storia ("Da qualche anno", comincia) di una "piccola cerchia, universitaria ed extra", presto battezzata "Circolo filologicolinguistico padovano". Non mi soffermerò su questa avventura che continua felicemente tuttora e che altri, specie gli amici padovani, potranno raccontare meglio di me, come anzi già hanno fatto. Sottolineo solo alcuni tratti, per cosi dire, morali, dichiarati preventivamente da Folena per il battesimo del primo Quaderno: passione («iniziativa appassionata e tenace»), apertura culturale ("anche al di là dell'ambito strettamente tecnico", confrontando "metodi e indirizzi diversi", "diversità dei punti di partenza", incontrando "studiosi lontani"), attenzione per i giovani (incoraggiandoli a "scegliere la propria via, in un contatto non solo interdisciplinare, com'è di moda dire oggi, ma personale e umano"), elogio della conversazione, dello scambio intellettuale, in un'epoca, scriveva "incline al formalismo logico e all'astrazione spesso dogmatica", in cui "si attribuisce tanto rilievo al processo della comunicazione e si finisce non di rado per perderne di vista l'oggetto, le cose e il loro valore": precisazione precoce contro i vari dogmatismi strutturalisti e formalisti che sarebbero venuti poi e che Folena presagiva già a quell'altezza cronologica.

Dopo un'ulteriore e rapidissima ricostruzione di come siano nati questo primo (nel 64-65) e il secondo quaderno tematico del Circolo (dedicato al teatro), entrambi testimoni della convergenza, a lui particolarmente cara, su uno stesso o analoghi oggetti da diversi "punti di partenza", Folena entra nelle Ricerche che introduce. In pochi paragrafi, dopo un franco cenno agli autori di cui (allora) poteva più essere lamentata la mancanza se si fosse ambito a un quadro esauriente della poesia novecentesca dopo mezzo secolo (Sbarbaro, Boine, Campana), Folena delimita il quadro temporale delle Ricerche ("l'entre deux guerres", anche se si comincia prima della Grande Guerra) e lo caratterizza in magistrale sintesi col percorso simmetricamente opposto di Ungaretti e Montale "fra tradizioni linguistiche indigene ed esperienze europee": Ungaretti, dopo essere espatriato verso Apollinaire e lo sperimentalismo prebellico francese, torna, sia pure via Mallarmé, a Petrarca e alla "tradizione linguistica indigena", mentre Montale, partito da un ripensamento libero (che "afferma e nega") di D’Annunzio, Pascoli e Gozzano, cioè "dalla poesia italiana vigente", procede verso i poeti metafisici inglesi, in particolare Eliot, "per interna necessaria convergenza". Due movimenti uguali e contrari con cui Folena disegna con acuta intelligenza la storia di due grandi poeti e due imprescindibili vicende culturali.

Poi Folena procede a fulminee annotazioni sulla poetica e sullo stile dei cinque poeti studiati nel volume (con particolare indugio su Rebora, che Contini aveva già autorevolmente rilanciato, ma che, certo, a quell'altezza cronologica meritava ancora qualche giustificazione), per arrivare a Montale, cui 
dedica indirettamente ma esplicitamente il volume, che usciva giusto in occasione dei suoi 70 anni. Per di più, essendo l'oggetto dello studio più ampio e importante del libro, quello di Pier Vincenzo Mengaldo, Montale gli offre alcuni spunti di teoria e critica letteraria su cui vorrei soffermarmi brevemente, partendo da una citazione:

Come ricorda Montale, citato qui in epigrafe da Mengaldo, "il linguaggio di un poeta è un linguaggio storicizzato, un rapporto. Vale in quanto si oppone o si differenzia da altri linguaggi". La storia di una lingua poetica si può fare solo risolvendo adeguatamente caso per caso questo rapporto differenziale, osservando dall'interno di essa le tradizioni che vi confluiscono in un raccourci pancronico e che vi assumono una configurazione del tutto nuova.

Cosi scrive Folena fissando nella dialettica tra cultura ricevuta e letteratura elaborata il punto migliore da cui osservare il linguaggio della poesia, il suo dipendere e dialogare con tradizioni e il suo concorrere a costituire delle tradizioni (che non vengono solo dal passato, ma sono anche esperienze condivise o assorbite dai contemporanei).

La dimensione diacronica è comunque essenziale per lo storico Folena, come per i suoi allievi e amici di quel primo Quaderno. La lingua di un poeta si coglie attraverso scarti e deviazioni: "ma relativamente a che cosa?". Qui Folena polemizza garbatamente con le teorie all'epoca ben rappresentate e sostenute della lingua poetica come differenza sì, ma da un'ipotetica langue de la tribu, dalla lingua comune: era l'idea che stava dietro le poche pagine che Tullio De Mauro aveva dedicato alla lingua poetica in una delle fitte appendici alla sua Storia linguistica dell'Italia unita (Laterza I963) e soprattutto era quella che sosteneva le acute ricerche (applicate proprio anche a Montale) di Luigi Rosiello (Struttura, uso e funzioni della lingua, Vallecchi 1965) e di quanti pensavano "di poter tradurre in quozienti numerici gli scarti del poeta", sbagliando, secondo Folena, perché "questi scarti qualitativi non sono mai misurabili quantitativamente" e perché non "sembra... metodologicamente legittimo paragonare un 'corpus' contestuale, di parole poetica realizzata, con un 'corpus' ipotetico di langue, ma se mai... parole di poeta con parole di poeta". Pur non negando, lo scrive esplicitamente, "il puntuale valore di verifica strumentale che le statistiche possono rivestire anche in questo campo", Folena sembra temere la perdita dello specifico letterario ove tradotto nella neutralità dei numeri.

Dunque, per Folena uno scarto contraddistingue la lingua della poesia, ma rispetto non tanto alla lingua comune e alla sua grammatica (perlomeno non solo, non esclusivamente: i plurali inconsueti o l'omissione dell'articolo davanti al nome nella poesia ermetica o la destrutturazione della coerenza testuale nella poesia odierna fanno anche questo; gli arcaismi e le varianti rare di forme comuni nella lirica dal Cinquecento all'Ottocento pure, anche se fino a tutto l'Ottocento la lingua comune era più virtuale e sperata che documentata e raggiunta), quanto ad altra, precedente lingua poetica, esattamente come (lo aveva insegnato Leo Spitzer, pur in chiave di identificazione della specificità psicologica più che storico-linguistica) un autore si costruiva il suo linguaggio deviando da altri stili e linguaggi.

Folena anticipa qui, di fatto, quello che, in quel volume del 1966, il lungo saggio di Mengaldo inaugurava: l'ampio e insuperato studio (cinque magistrali volumi dal I975 al 20I7) sulla Tradizione del Novecento, in cui il suo più importante e autorevole allievo e collega ha ricostruito continuità e discontinuità nella vicenda letteraria novecentesca, le riprese e i superamenti linguistici, il riuso e il diverso impiego che soprattutto la poesia ha sempre fatto di materiali preesistenti o paralleli: una prospettiva sulla lingua poetica che ne vede i movimenti, gli andirivieni, le negazioni e le riaffermazioni, in uno stretto connubio tra innovazione individuale (lo stile) e tradizione piu o meno prossima: «moto dentro moto», dice Folena per descrivere la tensione fra individualità e tradizioni 
poetiche, quando, guardando alle ricerche del Quaderno nel loro insieme, afferma, parando l'eventuale accusa di un eccesso di esprit de clocher con la doverosa citazione di studi precedenti e coevi come quelli di Contini, Avalle, Bonfiglioli e Barberi Squarotti, che esse "costituiscono il bilancio analitico complessivo più largo finora esperito" sul vivo patrimonio della lingua poetica novecentesca. La storia della lingua poetica era dunque possibile per Folena, ma iuxta propria principia e uno dei suoi strumenti, qui per la prima volta applicato alla poesia moderna, era la stilistica delle fonti: "il rapporto interno, dinamico con la tradizione, la stilistica delle fonti, si rivela il parametro più efficace per una storia concreta della lingua poetica, oltre che per la sua puntuale esegesi".

Qui si tocca con mano l'interpretazione autentica che Folena dà della lingua poetica, la cui dimensione storica e relativa interpretazione dinamica sono centrali per lui (e la sua scuola) e tali resteranno anche quando lo strutturalismo più totalitario spingerà la moda verso la sincronia e l'isolamento del testo. Se lo strutturalismo è poco o punto interessato alla storia, la storia della lingua la storia ce l'ha sempre avuta non solo nel nome, ma anche nel sangue: storia interna della lingua, dalla grammatica storica alle evoluzioni della grammatica e della norma (che Folena aveva magistralmente registrato nelle sue prefazioni alle antologie dei testi non toscani, specie quelli del Quattrocento) e storia esterna, dei suoi rapporti con la cultura e la società. Anche quando il suo oggetto è la lingua poetica, la storia non esce dal radar degli storici della lingua, e non solo la storia grammaticale e lessicale, ma anche quella artistica, sociale e politica.

La sensibilità alla storia, che è nel DNA della disciplina di cui Folena è stato uno dei primi grandi maestri, porterà a privilegiare, su quella degli strutturalisti ortodossi, la lezione dei formalisti russi, tra di essi in particolare di Yurij Tynjanov (Il problema del linguaggio poetico, trad. it. Il Saggiatore 1968), che aveva visto la dimensione diacronica della lingua della poesia segnata da antagonismi e superamenti, recuperi e opposizioni, insomma da una dialettica che realizzava a livello di sistema speciale (in una data epoca o scuola o autore) quello che la stilistica percepiva a livello di parole strettamente individuale (scarti, differenze all'interno dell'opera di un autore, di un testo). Non a caso, come esemplarmente dimostrato dalla vasta opera di Mengaldo, studio delle tradizioni linguistiche letterarie e analisi stilistica (e metrica) fanno spesso tutt'uno.

Questa sensibilità per la diacronia e quindi per la storicità degli eventi linguistici, anche poetici, ci ricorda che, se la nuova scienza letteraria aveva accantonato l'idealismo, non aveva del tutto buttato a mare il pur superato storicismo, ancorché in una lettura sua propria, del tutto indeterministica, e ovviamente completamente e deliberatamente "non organica" alla militanza politica (magari condivisa), come certuni allora avrebbero preteso. La laicità della critica è sempre stata un bene prezioso per i migliori intellettuali di sinistra.

C'è un altro tratto da segnalare nella prefazione a quel primo Quaderno; non troppo diverso da quelli sin qui enucleati. In premessa al libro forse più "tecnico" e specialistico sino ad allora uscito sulla poesia in quel momento contemporanea, Gianfranco Folena si muove anche da intellettuale militante. La specializzazione linguistica, con l'ampia e impeccabile documentazione che richiede per sua natura, non esclude, anzi dà sorprendente e onesto sostegno alla dimensione militante, cioè critica dell'interpretazione letteraria. Folena ne era ben consapevole e lo desiderava, da autentico intellettuale militante. Questa qualità e le due parole che la definiscono sono ben spiegate nella Presentazione di oltre mezzo secolo fa: intellettuale è illustrata dall'esplicita dichiarazione di apertura culturale che è alla base del progetto stesso del Circolo, di cui ho detto allinizio, e dalla mobilità e ampiezza del suo raggio d'osservazione: dai poeti italiani in esame nel volume ad Apollinaire, Mallarmé, Guillén, Eliot (non nominati per sfoggio, ma con concrete, idonee allegazioni), alle citazioni acute da Montaigne, da Sainte-Beuve e da Proust. Dell'autore della Recherche Folena riporta un brano da una lettera a 
un'amica, Mme Straus, che vale la pena rileggere, perché utile a corroborare l'idea della storia della lingua poetica per scarti, ritorni e superamenti che lo studioso era venuto delineando e che certo per questo motivo aveva qui recuperato quel cameo. All'amica che nel igo8 lo sollecitava a intervenire in difesa del francese, Proust scriveva:

La sola maniera di difendere la lingua consiste nell'attaccarla, ma sì, signora Straus! Perché la sua unità consiste solo di contrari neutralizzati, d'una immobilità apparente, che nasconde una vita vertiginosa e perpetua.

A ribadire l'apertura del raggio intellettuale del pensiero di Folena potremmo addurre anche, da quella presentazione, il suo non esibito ma istintivo, controllato e prorompente plurilinguismo tra tedesco e francese e l'emersione della ben nota competenza musicale (adoperata nel ritratto del linguaggio di Saba e nel ricordare "l'interpretazione enarmonica dell'universale Realtà" vagheggiata da Rebora, capace, scriveva, di una "soluzione antimelodica, carica di dissonanze, poliritmica e politonale"). Oggi, in epoca di specialismi magari stupendi ma troppo spesso chiusi, quando anche giovani, valenti studiosi si fanno un vanto di non studiare e leggere altro che quello che concerne il loro specifico oggetto di studio e, se medievisti, di ignorare la modernità, se modernisti le origini, quella di Folena è una lezione che sarebbe bene ripassare anche per questa ampiezza di orizzonti.

Intellettuale militante, dicevo, e vorrei riflettere anche sulla seconda parola del sintagma. Molto aiuterebbe a farlo la rilettura della rapida e appassionata analisi della poesia di Clemente Rebora, prete poeta di cui Folena sottolinea con compiacimento "l'attacco distruttivo e insieme ricostruttivo alla compagine della lingua poetica". Ma mi limiterò a leggere la conclusione della Presentazione, là dove Folena tira le somme del libro che lui stesso aveva voluto e sollecitato:

\begin{abstract}
Questo mi pare che costituisca l'apporto più positivo del presente libro: un contributo alla storicizzazione di questa esperienza di lingua poetica, che si allontana da noi, mentre la condizione della poesia nei confronti con la società è segnata da fratture assai più gravi di quelle che si verificarono all'inizio di questo movimento. Si allontana, eppure è cosi viva e attuale, e nel caso del nostro maggiore poeta ancora miracolosamente progrediente. Diceva Saint-Beuve che in periodi di anarchia o di confusione linguistica solo i poeti di genio si salvano "à la nage" e tutto il resto scompare. Non è mai stato vero, come nei nostri giorni, in cui la parola è "segnata dal commercio", senza scampo, che i poeti che si salvano portano in salvo la lingua.
\end{abstract}

C'è in queste parole un gesto di fiducia nella poesia anche come difesa e promozione di lingua che oggi quasi commuove e non è solo gesto emotivo o d'occasione, ma radicato in una cultura che è tanto linguistica quanto letteraria, conoscenza specialistica e passione civile. Oggi che i percorsi di ricerca della linguistica sembrano snobbare la letteratura (certo anche per il suo scarso peso nel bilancio linguistico del giorno) è bene ricordare la costitutiva letterarietà della storia della nostra lingua (mai dimenticare che quando ci inoltriamo in un ambiente usiamo un'invenzione di Dante come indovarsi o immiarsi): anche se ora meno percepibile, la lingua letteraria è tuttavia ancora ben salda nei fondamenti sempre vivi e operanti del sistema dell'italiano (e persino di certe sue regole, se non grammaticali, stilistiche). Non è un caso, allora, se il battesimo del primo Quaderno del suo Circolo ha per Folena un padrino ideale in Eugenio Montale, da cui cita la celebre confessione sulla sua lotta di poeta "per scavare un'altra dimensione nel nostro pesante linguaggio polisillabico". A Montale settantenne Folena fa omaggio di quello "xenion nato intorno a un tavolo per opera di giovani, a esprimergli la gratitudine per avere attaccato la lingua e lottato per la lingua, facendo emergere l'evidenza dal subbuglio".

C'è, certamente, in questa idea dialettica e inquieta della lingua e della poesia, la traccia di una cultura 
purtroppo ormai dimenticata, che non si accontentava dell'esistente e, per convinzione (filosofica) o per passione (politica), cercava il cambiamento e il miglioramento dell'umanità, ovunque, anche nell'arte, forse a partire dall'arte. Oggi che la (pre)potenza dell'esistente (economico, politico, mediatico...) sembra così incontenibile da indurre a non pensare più che quello che è potrebbe anche non essere o essere diverso (almeno in letteratura!), ritrovare una cosi autorevole testimonianza di quella perduta, operosa e profonda dimensione intellettuale e politica, protesa al mutamento e all'invenzione, alla ripresa e al superamento, fa sentire ancora più forte la nostalgia per persone come Folena che l'hanno tanto vigorosamente abitata.

Note:

* VV., Ricerche sulla lingua poetica contemporanea, Liviana Editrice, Padova 1966. Tutte le citazioni qui dalla Presentazione di Gianfranco Folena.

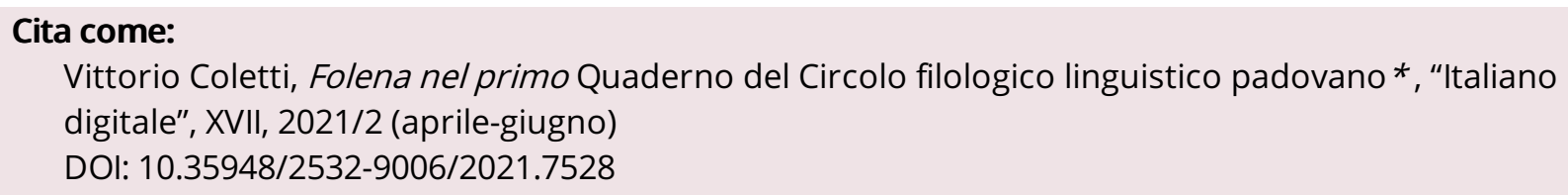

